\title{
Staphylococcal Colonisation of Intravenous Cannula and Antimicrobial Patterns of Isolated Staphylococcus Species
}

\author{
R. Mahto ${ }^{1}$, J. Amatya ${ }^{1}$, R. Amatya ${ }^{2}$, and D. Adhikari ${ }^{1}$ \\ ${ }^{1}$ Kathmandu College of Science and Technology, Kathmandu \\ ${ }^{2}$ Nepal Medical College, Jorpati, Kathmandu \\ e-mail: mahato_rameshwor@yahoo.com
}

\begin{abstract}
Cannula-related infections are amongst the most important nosocomial infections. Staphylococcus aureus and coagulase negative Staphylococci (CONS) are the most frequent causes of colonizing the intravenous cannula through which infection may occur. The aim of the study was to isolate the colonized staphylococci and to study the antimicrobial sensitivity pattern against them. Cultures of cannula tips were raised by semi-quantitative method. Antibiotic susceptibility test was performed by Kirby Bauer disc diffusion method as per The Clinical and Laboratory Standard Institute (CLSI) guidelines. Coagulase positive Staphylococcus was confirmed by coagulase slide test followed by tube test. CONS were distinguished by novobiocin sensitivity test. Out of 200 samples of cannula tips collected and processed, $45(22.5 \%)$ isolates were S. aureus, $15(7.5 \%)$ were S. epidermidis and $16(8 \%)$ were other CONS. The highest occurance (30.7\%) of S. aureus and the CONS (18.4\%) was found in the nephrology ward and the surgical ward respectively. All the 76 isolates were tested for antimicrobial susceptibility pattern. Novobiocin was used to differentiate $S$. epidermidis from other CONS. All isolates were sensitive to ampicillin and ofloxacin. Out of total, $46.7 \%$ isolates of S. aureus and $12.9 \%$ isolates of CONS were multidrug resistant; $35.5 \%$ and $20 \%$ isolates of S. aureus were oxacillin (methicillin) resistant and vancomycin resistant respectively. Four $(12.9 \%)$ isolates of CONS [2(13.3\%) of other CONS and 2(12.5\%) of S. epidermidis] were oxacillin (methicillin) and vancomycin resistant. In our study, vancomycin resistant staphylococci were found and it was concluded that the precautions for inserting intravenous cannula is necessary as different kinds of pathogens may colonize such devices.
\end{abstract}

Key words: CONS, methicillin resistant, vancomycin resistant

\section{Introduction}

The use of indwelling cannula for reliable intravascular access is an essential feature of modern health care. Insertion of intra venous (IV) cannula allows continuous and painless access to the circulation for administration of fluids and electrolytes, medications, blood products and nutritional support. Cannularelated infections are amongst the most important nosocomial infections. Between $5 \%$ and $25 \%$ of IV devices are found to be colonized by skin organisms (Pratt et al. 2001).

Infection of indwelling intravenous cannula occurs from two routes. First, endogenous skin flora at the insertion site migrate along the external surface of the cannula and colonizes the intravenous tip. Organisms may be acquired from patients' micro flora, hands of staff, contaminated disinfectants, hub and fluids, ward air, etc.. Second, pathogens from hub contamination move along the internal surface of the cannula and colonize the lumen. The incidence of infection is related to the length of time the cannula remains in situ. The cannula may be the source of a bacteraemia (Gutierrez et al. 1992). Infection of IV cannula may lead to widespread dissemination of infection. More usually the patients develope fever and may become generally unwell. Localized infection can occur at the insertion site and subcutaneous track of the device (Elliott et al. 1994). Clinical signs of infection include 
erythema, exudate formation, oedema and thrombophlebitis. Colonizing organisms may include CONS, S. aureus, Enterobacteriaceae, Pseudomonads, Enterococci, Corynebacterium species, Streptococci, Bacillus species, Candida albicans and other yeasts, Aspergillus species, Fusarium species, Malassezia furfur, etc (Maki et al. 1992).

CONS and S. aureus are the most frequent causes of cannula-related infections. They can produce extracellular slime that facilitates adherence and may limit the access of antibiotics, and may reduce the host's inflammatory response (Maki et al. 1992). Over the last decade, methicillin resistant $S$. aureus (MRSA) strains have become endemic in hospitals worldwide. In 1980s, because of widespread occurrence of MRSA, empiric therapy for staphylococcal infections was changed to vancomycin in many health care institutions. Thus, the early 1990s have shown a discernible increase in vancomycin use that eventually lead to the emergence of strains of $S$. aureus and other species of staphylococci with decreased susceptibility to vancomycin (Tenover et al. 2001).

\section{Methodology}

This descriptive study was carried out prospectively at KIST Medical College Teaching Hospital during January to August 2011. Study was conducted on patients admitted in surgical ward (SW), medical ward (MW), orthopedic ward (OW) and nephrology ward
(NW). A total of 200 samples (cannula tips) from patients who were inserted intravenous cannula were included in the study. The samples were processed according to the semi quantitative culture method. Forceps were dipped in $95 \%$ alcohol and were flame sterilized and allowed to cool. Slight pressure was used to roll the tips back and forth across the blood agar surface at least four times and the plates were incubated for at least 72 hours at $37^{\circ} \mathrm{C}$. Microbial growths were observed each day and the colony were counted. If the colony count was less than 15 then it was considered as no growth. Gram staining, catalase test, oxidase test and coagulase test (slide and tube test) were done to confirm the Staphylococcus species. Ampicillin, erythromycin, gentamycin, oxacillin, ofloxacin, penicillin and vancomycin were used for the antibiotic susceptibility test (AST). Novobiocin was used to differentiate S. epidermidis (sensitive) from other CONS. Statistical analysis was done by using Statistical Package for the Social Sciences version 18.0.

\section{Results and Discussion}

A total of 200 samples of cannula tips were collected and processed, out of which $109(54.5 \%)$ samples showed growth on culture; 45 (22.5\%) isolates were S. aureus, $15(7.5 \%)$ were S. epidermidis and $16(8 \%)$ were other CONS (Total CONS:15.5\%). The patients having the age group 60 year to more consisted of the higher percentage of $S$. aureus. More percentage of CONS was seen in the age group of 15 to 60 year (Table 1).

Table 1. Agewise distribution of organisms

\begin{tabular}{l|l|l|ll|l}
\hline \multicolumn{2}{c}{} & \multicolumn{3}{c}{ Organism } \\
\cline { 3 - 5 } $\begin{array}{l}\text { Age group } \\
\text { (year) }\end{array}$ & $15-60$ & S. aureus & Other CONS & S. epidermidis \\
& $(\mathrm{n}=140)$ & Count & 31 & 13 & 14 \\
& $\geq 60$ & Count & $22.1 \%$ & $9.3 \%$ & $10 \%$ \\
Total $(\mathrm{n}=200)$ & $(\mathrm{n}=60)$ & \% of Total & $23.3 \%$ & $2.3 \%$ & 2 \\
& & Count & 45 & 15 & $3.3 \%$ \\
& & \% of Total & $22.5 \%$ & $7.5 \%$ & 16 \\
\hline
\end{tabular}

Table 2. Wardwise distribution of isolated staphylococci

\begin{tabular}{|c|c|c|c|c|}
\hline \multirow[b]{2}{*}{ Ward } & \multicolumn{3}{|c|}{ Organisms } & \multirow[b]{2}{*}{ Total } \\
\hline & S. aureus & Other CONS & S. epidermidis & \\
\hline $\operatorname{MW}(n=76)$ & $\begin{array}{l}14 \\
18.4 \%\end{array}$ & $\begin{array}{l}6 \\
7.9 \%\end{array}$ & $\begin{array}{l}5 \\
6.6 \%\end{array}$ & $\begin{array}{l}25 \\
32.9 \%\end{array}$ \\
\hline SW & 16 & 7 & 5 & 28 \\
\hline$(n=65)$ & $24.6 \%$ & $10.7 \%$ & $7.7 \%$ & $43 \%$ \\
\hline $\mathrm{NW}(\mathrm{n}=26)$ & 8 & 1 & 3 & 12 \\
\hline $\mathrm{OW}(\mathrm{n}=33)$ & $\begin{array}{l}30.7 \% \\
7 \\
21.21\end{array}$ & $\begin{array}{l}3.8 \% \\
1 \\
3.03 \%\end{array}$ & $\begin{array}{l}11.5 \% \\
3 \\
9.09 \%\end{array}$ & $\begin{array}{l}46 \% \\
11 \\
33.33 \%\end{array}$ \\
\hline
\end{tabular}


The highest percentages ( $46 \%$ ) of staphylococci were found in the NW and the lowest percentages of organism $(32.9 \%)$ were found in the MW (Table 2). Out of 45 isolates of $S$. aureus, $35.5 \%$ were oxacillin (methicillin) resistant, and 20\% were vancomycin resistant. Similarly, out of 31 CONS, $4(12.9 \%)$ isolates [2(13.3\%) of other CONS and $2(12.5 \%)$ of $S$. epidermidis] were oxacillin (methicillin) resistant and vancomycin resistant. All isolates of staphylococci were sensitive to ampicillin and ofloxacin (Table 3).

Table 3. Antibiotic susceptibility pattern for organisms

\begin{tabular}{|c|c|c|c|c|c|c|}
\hline \multirow[t]{2}{*}{ Antibiotics } & \multicolumn{2}{|c|}{$\begin{array}{l}\text { Organism } \\
\text { S. } \text { aureus }(\mathrm{n}=45)\end{array}$} & \multicolumn{2}{|c|}{ Other CONS $(n=15)$} & \multicolumn{2}{|c|}{ S. epidermidis $(\mathrm{n}=16)$} \\
\hline & Sensitive & resistant & Sensitive & resistant & Sensitive & Resistant \\
\hline Ampicillin (AM) & $\begin{array}{l}45 \\
(100 \%)\end{array}$ & 0 & $\begin{array}{l}15 \\
(100 \%)\end{array}$ & 0 & $\begin{array}{l}16 \\
(100 \%)\end{array}$ & 0 \\
\hline Erythromycin (E) & $\begin{array}{l}30 \\
(66.7 \%)\end{array}$ & $\begin{array}{l}15 \\
(33.3 \%)\end{array}$ & $\begin{array}{l}13 \\
(86.7 \%)\end{array}$ & $\begin{array}{l}2 \\
(13.3 \%)\end{array}$ & $\begin{array}{l}14 \\
(87.5 \%)\end{array}$ & $\begin{array}{l}2 \\
(12.5 \%)\end{array}$ \\
\hline Gentamycin(Gen) & $\begin{array}{l}43 \\
(95.6 \%)\end{array}$ & $\begin{array}{l}2 \\
(4.4 \%)\end{array}$ & $\begin{array}{l}14 \\
(93.3 \%)\end{array}$ & $1(6.7 \%)$ & $\begin{array}{l}16 \\
(100 \%)\end{array}$ & 0 \\
\hline Ofloxacin (Of) & $\begin{array}{l}45 \\
(100 \%)\end{array}$ & 0 & $\begin{array}{l}15 \\
(100 \%)\end{array}$ & 0 & $\begin{array}{l}16 \\
(100 \%)\end{array}$ & 0 \\
\hline Oxacillin (Ox) & $\begin{array}{l}29 \\
(64.4 \%)\end{array}$ & $\begin{array}{l}16 \\
(35.5 \%)\end{array}$ & $\begin{array}{l}13 \\
(86.7 \%)\end{array}$ & $\begin{array}{l}2 \\
(13.3 \%)\end{array}$ & $14 \quad(87.5 \%$ & $2(12.5 \%)$ \\
\hline Vancomycin (Va) & $\begin{array}{l}36 \\
(80 \%)\end{array}$ & $\begin{array}{l}9 \\
(20 \%)\end{array}$ & $\begin{array}{l}13 \\
(86.7 \%)\end{array}$ & $\begin{array}{l}2 \\
(13.3 \%)\end{array}$ & $\begin{array}{l}14 \\
(87.5 \%)\end{array}$ & $\begin{array}{l}2 \\
(12.5 \%)\end{array}$ \\
\hline Penicillin (P) & $\begin{array}{l}32 \\
(71.1 \%)\end{array}$ & $\begin{array}{l}13 \\
(28.9 \%)\end{array}$ & $\begin{array}{l}14 \\
(93.3 \%)\end{array}$ & $\begin{array}{l}1 \\
(6.7 \%)\end{array}$ & $\begin{array}{l}14 \\
(87.5 \%)\end{array}$ & $\begin{array}{l}2 \\
(12.5 \%)\end{array}$ \\
\hline
\end{tabular}

In the present study, among the 200 samples of cannula tip subjected to culture, only 109 (54.5\%) showed growth on culture, $45(22.5 \%)$ were S. aureus, and 31 $(15.5 \%)$ were coagulase negative staphylococci. The overall infection rates from world literature for intravascular cannula were $3.8 \%$ to $57 \%$, (Samsoondar et al. 1985) and the overall colonization rate in this study was $54.5 \%$ which validated the study and implied that colonization of organism in intravenous cannula in the hospitalized patients cannot be neglected just as the contamination since it causes phlebitis and rarely blood stream infection. The Centre for Disease Control and Prevention estimates that there are 250,000 hospital-acquired bloodstream infections per year, with the vast majority of these associated with central venous catheters (Rizzo et al., 2005). In the study done in India, $21.3 \%$ CONS were isolated whereas only $12.5 \%$ S. aureus were isolated (Parameswaran et al. 2011). In the study done in Egypt, $35 \%$ isolated organisms were CONS and $8.3 \%$ were $S$ aureus (Awad et al. 2011). One hundred and fortyeight organisms were isolated as the cause of cannula colonization. The most commonly isolated organism was $S$. aureus $(\mathrm{n}=52$, i.e.37.1\%) and S. epidermidis ( $\mathrm{n}$ $=26$, i.e. $18.6 \%)($ Serkan et al. 2003). All these studies from different parts of the worlds have suggested that
CONS and S. aureus were the most isolated organisms found in the cannula tips.

The age group of more than 60 year consisted of the highest percentage of $S$. aureus. Infection rates are higher among patients with increased susceptibility because of old age, deterioration of the immune system and the severity of the underlying disease. More percentage of CONS was seen in the age group of 15 to 60 . This suggests that the active and younger aged group also cannot be neglected for the staphylococcal colonization.

The highest percentages ( $46 \%$ ) of staphylococci were found in the nephrology ward. In the study done in intensive care unit, $61.5 \%$ S. epidermidis and $8.3 \%$ S. aureus (Dimick et al. 2001) were found. Nephrology ward is related to the patients of kidney and urinary tract infections and staphylococci were also the predominating organisms there, which may be the reason of presence of higher staphylococci in the nephrology ward.

In the present study, all the 76 isolates of staphylococci were sensitive to ampicillin and ofloxacin. Higher percentage $(33.33 \%)$ of $S$. aureus was oxacillin 
resistant. Oxacillin resistant means they are automatically resistant to methicillin and hence called MRSA. Similarly, 3(18.8\%) isolates of S. epidermidis and $2(13.3 \%)$ isolates of other CONS were methicillin resistant. Resistance to methicillin among S. aureus increased from 1.5 per cent in 1986 to $31.2 \%$ in 2002. With regard to CONS, the increase in resistance to methicillin was even greater, reaching to $61.3 \%$ (Cuevas et al. 2004).

The present study also shows that there is a significant rise of reduced susceptibility of vancomycin. $20 \%$ isolates of S. aureus, $12.5 \%$ isolates of S. epidermidis and $13.3 \%$ of other CONS were vancomycin resistant. Though first case of vancomycin resistant $S$. aureus (VRSA) was reported in 2002 in USA (Centers for Disease Control and Prevention, 2002) but few other countries have reported the reduced susceptibility of S. aureus and CONS against vancomycin. Recently (Palazzo et al. 2005) have also reported some vancomycin resistant strains of CONS in Brazil. More recently Bataineh has reported VRSA strains from Jordan (Bataineh 2006). The current vancomycin resistant staphylococci in hospital as well as in community are alarming situations to the clinicians. The development of antibiotic resistance in developing countries like ours seems to be very much related to the irrational antibiotic usage due to its easy availability at the drug stores without prescription, injudicious use in hospitals and uncontrolled use in agriculture, animal husbandry and fisheries (Holloway 2000). Vancomycin is currently the main antimicrobial agent available to treat life-threatening infections with MRSA. Widespread use of vancomycin to treat infections caused by MRSA and other gram-positive cocci has led to the emergence of vancomycin resistance. The large scale of development and subsequent spread of resistance to vancomycin has been perceived as a fearsome threat to the already challenging therapy of MRSA.

The method which we used to culture the cannula tip (semi quantitative method) has a limitation in that it can take samples only from the external surfaces of cannula and may not retrieve organisms from the internal surfaces of the cannula (Maki et al. 1992). The semi quantitative cannula segment culture is one of the least expensive tests for a clinical microbiology laboratory to perform and the most frequently used method in hospital laboratory.
Colonization of Staphylococcus species in the intravenous cannula is still an important and ongoing problem in medical practice. This study highlights the vancomycin resistance of staphylococci with methicillin resistant staphylococci. The resident or transient cutaneous flora are the source of infection. Multiple factors, including the infusate and the duration of cannulation, the length of cannulation, level of asepsis at insertion contribute to the development of intravenous cannula-related colonization and infection. In addition to proper insertion and good nursing care, the avoidance of the risk factors contributing colonization in cannula will lead to a lower incidence of cannula associated colonization and infection.

\section{Acknowledgements}

We gratefully acknowledge the support provided by the KIST Medical College Teaching Hospital, Imadol, Lalitpur for this study.

\section{References}

Awad, S.A., A.H. El-Soussi, M.A. Sultan, M. El-Farrash and N. Tantawy. 2011. Effect of intravenous catheter placement on venous pressure reading and the risk of complications in critically Ill patients. J. Amer. Sc. 7(10):645-655.

Bataineh, A.B. 2006. Resistance of Staphylococcus aureus to Vancomycin in Zarqa, Jordan. Pak. J. Med. Sci. 22:144-148.

Centers for Disease Control and Prevention. 1997. Staphylococcus aureus with reduced susceptibility to vancomycin United States. Morb Mortal Wkly Rep: 46. $765 \mathrm{pp}$.

Cuevas, O., E. Cercenado, A. Vindel, J. Guinea, M. SanchezConde and M. Sanchez-Somolinos. 2004. Evolution of the antimicrobial resistance of Staphylococcus spp. in Spain: Five nationwide prevalence studies 1986 to 2002. Antimicrob. Chemother. 48:4240-4245.

Dimick, J.B., R.K. Pelz, R. Consunji, S.M. Swoboda, C.W. Hendrix and P.A. Lipsett. 2001. Increased resource use associated with catheter-related blood stream infection in the surgical intensive care unit. Arch. Surg. 136: 229-234.

Elliott, T.S.J., M.H. Faroqui, R.F. Armstrong and G.C. Hanson. 1994. Guidelines for good practice in central venous catheterization. J Hosp Infect. 28:1163-1176.

Goldmann, D.A. and G.B. Pier. 1993. Pathogenesis of infections related to intravascular catheterization. Clin. Microbiol. Rev. 6:176-192. 
R. Mahto/Staphylococcal Colonisation of Intravenous .....

Gutierrez, J., A. de la Higuera and G. Piedrola. 1992. Microbiological techniques for the diagnosis of intravascular catheter associated infections. Med. Microbiol. Lett: 1:243-250.

Holloway K. 2000. Antimicrobial resistance: the facts. Essential Drug Monitor WHO 29: 7-8.

Maki D. G, 1992. Infections due to Infusion Therapy. In: Hospital infections, $3^{\text {rd }}$ ed. (Eds. J.V. Bennett and P.S. Brachman). Little Brown \& Co., Boston, pp. 849898.

Palazzo, I.C.V., M.L.C. Araujo and A.L.C. Darini. 2005. First report of vancomycin-resistant staphylococci isolated from healthy carriers in Brazil. J. Clin. Microbiol. 43:179-185.

Parameswaran, R., J.B. Sherchan, M.D. Varma, C. Mukhopadhyay and S. Vidyasagar. 2011. Intravascular catheter-related infections in an Indian tertiary care hospital. J. Infect. Dev. Ctries. 5(6):452-458

Pratt, R.J., C. Pellowe, H.P. Loveday, N. Robinson, G.W. Smith and S. Barrett. 2001. The epic project: developing national evidence-based guidelines for preventing healthcare associated infections. Phase I: Guidelines for preventing hospital-acquired infections, Department of Health (England). J. Hosp. Infect. 47:73-82.

Rizzo, A.J. and C. Gunarrson. 2005. The effects of laproscopic surgery and nosocomial infections on the cost care: Evidence from 3 common surgical procedures. MEDLINE 15:191-194.

Samsoondar, W., J.B. Freeman, I. Coltish and C. Oxley. 1985. Colonization of intravascular cannulae in the intensive care unit. Am. J. Surg. 149:730-732.

Serkan oncu, Halit ozsut, Ayse Yildirim, Pinar Ay, Nahit Çakar, Haluk Eraksoy and Semra Calangu . 2003. Central venous catheter related infections: Risk factors and the effect of glycopeptide antibiotics Annals of Clinical Microbiology and Antimicrobials 2:3.

Tenover, F.C., J.W. Biddle and M.V. Lancaster. 2001. Increasing resistance to vancomycin and other glycopeptides in Staphylococcus aureus. Emerg. Inf. Di.7:327-332. 
Nepal Journal of Science and Technology Vol. 14, No. 2 (2013) 131-136 